\title{
MANEJO INTEGRAL DE UN PACIENTE NIÑO CON PÉRDIDA PREMATURA DE MOLARES DECIDUOS: REPORTE DE UN CASO
}

${ }^{1}$ Luswin Flórez M., ${ }^{2}$ Denisse Sarmiento 0.

${ }^{1}$ Estudiante IX semestre, Facultad de Odontología, U. Santo Tomás, ${ }^{2}$ Odontóloga, U. Santo Tomás.

Autor responsable de correspondencia: Luswin Flórez M.

Correo electrónico: lupinfmt@hotmail.com

\begin{abstract}
RESUMEN
Una gran variedad de aparatología fija y removible se ha sugerido para la distalización de molares, cuando existe falta de espacio para la erupción de dientes permanentes debido a la perdida prematura de los molares deciduos. Una alternativa práctica en el manejo interceptivo de este tipo de pacientes, la representa el arco transpalatino, cuyo uso esta dado como mantenedor de espacio en la zona posterior de maxilar, aunque en casos especiales puede aplicarse como reganador de espacio con muy buenos resultados. El objetivo de este artículo es presentar el caso de una paciente en la que fue necesario usar una barra transpalatina. [Flórez L, Sarmiento D. Manejo integral de un paciente niño con pérdida prematura de molares deciduos: Reporte de un caso. Ustasalud Odontología 2004; 3: 61 - 65]
\end{abstract}

Palabras clave: Pérdida prematura, barra transpalatina.

\section{INTEGRAL MANEGEMENT OF A CHILD WITH PREMATURE LOSS OF PRIMARY MOLARS}

\begin{abstract}
A great variety of fixed and removable appliances have been suggested for the distalization of molars, when space lack exists for the eruption of permanent teeth due to the premature lost of the primary molars. An alternative in the interceptive treatment of this type of patient is the transpalatal bar. Its use is like space retainer in the posterior area of maxillary, although in special cases it can be applied as space maintainer with very good results. The objective of this article is to present the case of a patient in whom it was necessary to use a transpalatal bar.
\end{abstract}

Key words: Premature loss, transpalatal bar.

Recibido para publicación: 20 de febrero de 2004. Aceptado para publicación: 30 de mayo de 2004

\section{INTRODUCCIÓN}

Se ha descrito ampliamente la pérdida temprana de dientes temporales asociada a aspectos como trauma dental, reabsorción radicular prematura o caries lo que puede generar maloclusiones. ${ }^{1,2}$

La principal secuela de la pérdida temprana de molares deciduos en el arco superior es la migración mesial de los primeros molares permanentes, ${ }^{2}$ presentándose relaciones atípicas entre los labios y los dientes que se caracterizan principalmente por desarrollo de maloclusiones, como es el caso de una Clase II División I. ${ }^{2-7}$

"El mantenimiento de este espacio en el arco puede ser suficiente para resolver la pérdida temprana del segundo molar temporal y/o su sucedáneo", ${ }^{6}$ pero cuando el espacio perdido por la migración es considerable, se puede planear su eventual recuperación. Estos dos manejos pueden realizarse mediante aparatología ortopédica fija 0 removible o una combinación de los dos tipos, ${ }^{2-8}$ previo un análisis completo del caso, el cual incluye análisis cefalométrico, análisis de dentición mixta y de espacios. ${ }^{9,10}$

La barra transpalatina se presenta como una alternativa en el manejo ortodóntico de estos pacientes; es un aparato fijo, que está conformado por dos bandas ubicadas en los primeros molares permanentes, unidas con un arco que transcurre transversalmente por la bóveda palatina a $2 \mathrm{~mm}$; el arco en la mitad lleva un ansa la cual permite realizar la activación al aparato., ${ }^{3,4,6}$

El arco transpalatino como mantenedor o reganador de espacio, limita los desplazamientos mesial de los molares permanentes, ante la pérdida temprana de molares 
deciduos, impidiendo su rotación y evitando la pérdida de longitud en el arco., ${ }^{4,6}$

Su principal indicación como reganador de espacio está dada, cuando uno de los dos lados del arco dental se encuentra intacto y faltan varios dientes primarios en la otra hemiarcada. El rígido anclaje del lado intacto le proporciona la estabilidad necesaria y sirve como eje para aplicar las fuerzas de distalización al molar, consiguiendo así el espacio necesario, la literatura reporta que éste puede ser de $3 \mathrm{~mm} .{ }^{1,4,8}$ De esta manera el arco transpalatino puede representar una buena alternativa de tratamiento, en pacientes con maloclusión localizada a causa de premolares impactados. ${ }^{2,5}$

El propósito de este artículo es presentar un caso clínico con el manejo integral de una paciente, que presenta pérdida de longitud en el arco dentario superior a causa de caries extensas.

\section{REPORTE DE CASO}

Paciente de sexo femenino, 9 años de edad, llega a las Clínicas Odontológicas de la Universidad Santo Tomás (USTA) en Floridablanca en febrero 2003. La mamá de la paciente estaba interesada en iniciarle el tratamiento odontológico. El motivo de consulta fue "la niña tiene los dientecitos dañados y se le están torciendo". Como antecedente médico familiar presentaba episodios de epilepsia. No se encontró otra alteración sistémica y no reportó antecedentes odontológicos personales.

Se le realizó el análisis facial, funcional, radiográfico y dental y se concluyeron los siguientes diagnósticos:

Diagnóstico funcional: Respiración mixta e incompetencia labial, hipotonicidad e interposición lingual, masticación unilateral izquierda.

Diagnóstico facial:Mesocefálico, mesoprósopo, perfil convexo, selle labial forzado, tercio medio e inferior aumentado, proquelia de labio inferior, surco mentolabial ausente, línea de sonrisa inferior y nariz pequeña (Figura 1,2 y 3$)$.

Diagnóstico dental: Mordida abierta anterior, relación molar de Angle izquierda: Clase I; derecha: Clase II. Caries OMV en el 55, caries OD en el 54, caries ODV en el 74, periodontitis periapical crónica no supurativa en el 65, caries secundaria en el 84. En el análisis de Moyers se encontró falta de espacio en el arco dentario superior e inferior. $^{9}$

En el examen radiográfico dental se observó la presencia de radiolucidez en los molares deciduos, compatible con caries dental; no se observaron alteraciones en los gérmenes dentarios, la secuencia de erupción cronológica era normal y a nivel óseo no presentó ninguna alteración (Figura 4).

\section{Diagnóstico esquelético:}

Con base en el análisis cefalómetrico de Mcnamara y Steiner, ${ }^{11,12}$ se determino una Clase II esquelética, macrognatismo y prognatismo del maxilar superior. Retrognatismo del maxilar inferior, protrusión de incisivos superiores e inferiores, tendencia a mordida abierta anterior patrón de crecimiento vertical y tercio inferior aumentado.

En la fase preventiva del plan de tratamiento se proyectó, control de placa, profilaxis, fisioterapia oral; en la ambientación dental se planeó las obturación del 84 .

En la fase de adecuación luego de llegar a un diagnóstico definitivo en el caso del 55, 54 y 65, se decidió realizar las exodoncias de estos molares. Posteriormente, se evaluó la posibilidad de colocar un mantenedor de espacio. Para la elección del tipo de mantenedor de espacio a usar en el manejo del paciente se tuvieron en cuenta las indicaciones, ventajas y desventajas de los aparatos. Se eligió como mantenedor de espacio, el arco transpalatino.,

Luego de cementar el arco en boca con ionómero de vidrio y realizarle los ajustes pertinentes se procedió a realizar las exodoncias. Se programó el primer control las dos semanas, cita en la cual se observó la evolución satisfactoria en la cicatrización. Posteriormente, se programaron controles cada mes. En agosto del mismo año fue remitida a la clínica de odontopediatría de VIII semestre de la USTA. Se le realizó la reevaluación clínica y se determinó que el 25 se estaba impactando. Se procedió a quitar el aparato y a seleccionar un reganador de espacio de acuerdo a las condiciones del paciente.

Se consideró oportuno seguir el tratamiento con la barra transpalatina; no sólo por cumplir con las indicaciones que se requerían en este manejo si no por que de esta manera se reducirían los costos y el tiempo de fabricación de un nuevo aparato. 


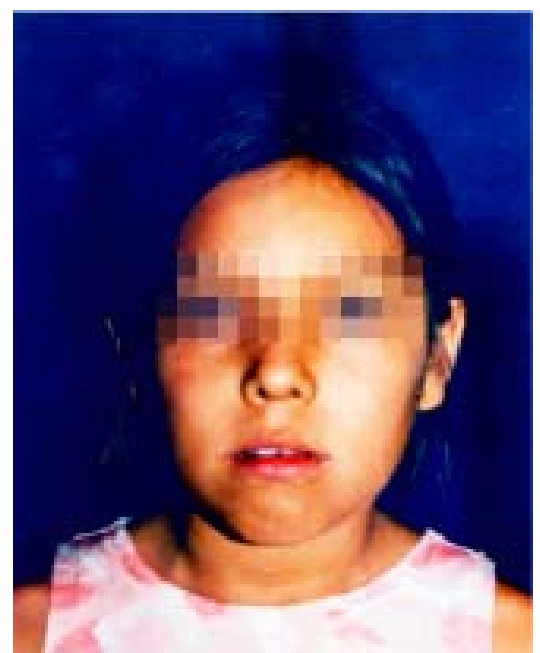

Foto 1. Frente.

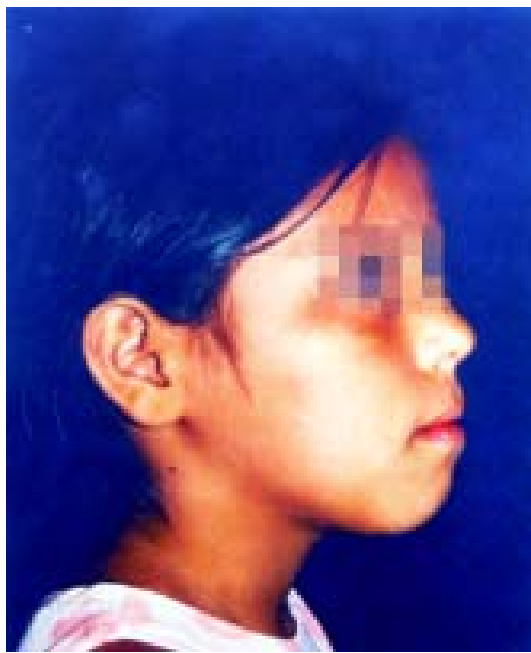

Foto 2. Perfil.

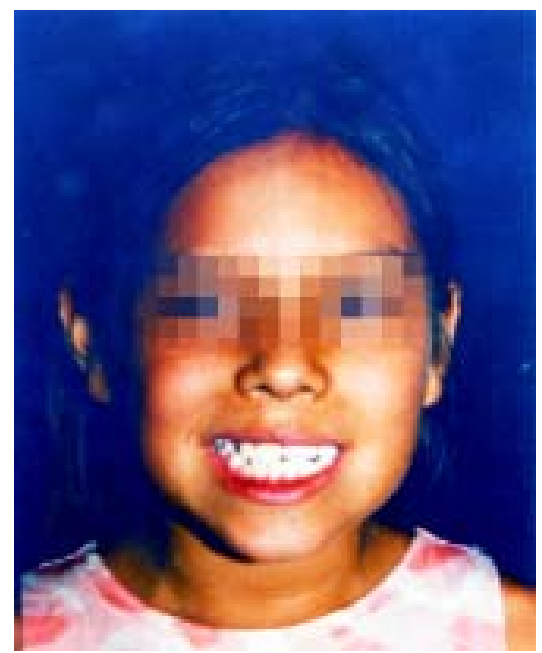

Foto 3. Sonrisa.
Se activó el arco con el objeto de lograr la distalización del 26. Se colocó el dispositivo en boca y se programaron las activaciones cada dos semanas. Se observó una mejoría en las condiciones bucales de la paciente.

\section{DISCUSIóN}

La literatura ha descrito ampliamente los motivos que provocan la pérdida prematura de molares deciduos y ha llegado a la conclusión que la caries es la principal causa; ${ }^{2,7}$ esta pérdida temprana de los molares temporales es más frecuente en maxilar inferior. ${ }^{1}$ Van der Linden, describe que en comparación con el maxilar inferior, el arco superior es el que presenta mayores secuelas. ${ }^{2}$

Las consecuencias de la terapia con exodoncias son muy discutidas. Pueden ser la causa del desarrollo de maloclusiones, que pueden inducir a un desequilibrio a nivel de los sistemas respiratorio y masticatorio. ${ }^{6,14}$

Se ha propuesto una gran variedad de tratamientos y de aparatología para tratar la pérdida de espacio en el segmento posterior del maxilar superior. Estos van desde aparatos tradicionales de mantenimiento de espacio 0 de distalización, hasta técnicas de expansión transversal. $^{1,4,5}$

Esta aparatología ortodóntica, básicamente, se clasifica en dos grupos: Aparatos removibles y fijos. Su elección debe depender de aspectos como el diagnóstico, los costos y la colaboración del paciente; su uso debe estar limitado por un conocimiento amplio de los inconvenientes y de las consecuencias indeseables que éstos producen. ${ }^{1,5}, 6,13,14$

El mayor inconveniente de la terapia con aparatología removible, es que su éxito depende de la colaboración del paciente. En el caso de los cráneomaxilares, se ha observado que un alto porcentaje de los pacientes no lo usan durante el tiempo indicado por el especialista. ${ }^{8,13,15}$

Se han ideado diversos tipos de aparatos para producir el movimiento de distalización molar. En este grupo de aparatos se encuentran los elementos magnéticos. Su gran desventaja es el alto costo y, aún no se han realizado estudios a largo plazo acerca de las consecuencias biológicas de su uso. Por otra parte su efectividad clínica, y la relación con respecto a otras terapias mecánicas es también objeto de discusión, ${ }^{1,16}$ aunque Steger y Blechman describen rápidos resultados con el uso de esta técnica, controlando el anclaje con aparatos convencionales como el Botón de Nance.,13

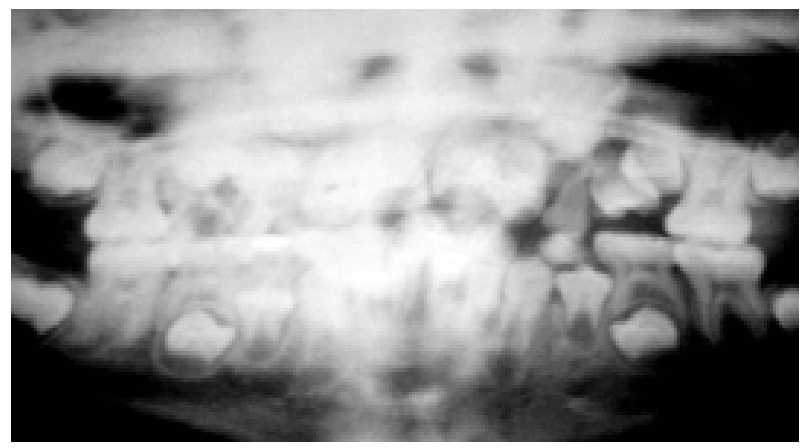

Foto 4. Radiografía panorámica. 
Reiner, en 1992, presentó un estudio realizado en 20 pacientes, con clase II unilateral en el que se empleó un Botón de Nance modificado, y reportó un ligero aumento de la altura facial inferior debido a la rotación vertical del maxilar inferior, después de haber conseguido una relación molar de Angle clase I. $^{13}$

Uno de los aparatos distalizadores usados más recientemente y que, actualmente, se encuentra en evolución es el Firss Class. Al parecer este aparato produce rápidos resultados incluso en presencia del segundo molar permanente. Sin embargo, por ser un aparato relativamente nuevo en el mercado existen pocos estudios con relación a los efectos dentales y esqueléticos que produce. ${ }^{1}$

Problemas como la inclinación distal y extrusión del primer molar superior, la rotación posterior del maxilar inferior, así como vestibuloversión de los incisivos y el aumento de la altura facial anterior, entre otros, son los mayores inconvenientes biológicos de la aparatología fija. Aunque las respuestas a este tipo de terapia dependen del patrón de crecimiento de cada paciente. ${ }^{1,6,13}$

Una alternativa efectiva para distalar molares superiores y conseguir la longitud de arco perdida la presenta el arco transpalatino; como aparato distalizador, ofrece una opción acertada; sólo en casos donde la pérdida de espacio no sea excesiva y se tenga un lado intacto del arco dentario que proporcione el anclaje requerido para la activación del aparato. ${ }^{6}$

En el manejo dado a este paciente se decidió utilizar el arco transpalatino para la distalización molar, considerando que la pérdida de espacio no era excesiva (3 mm). También se consideró la reducción en el tiempo de fabricación del aparato y la condición socio económica de la paciente. ${ }^{16}$

Aunque existe una gran variedad de aparatología para el manejo ortodóntico de la pérdida prematura de dientes deciduos, el fácil manejo y la aplicación terapéutica del arco transpalatino, así como su efectividad y sus bajos costos ofrece al odontólogo integral y al especialista una buena opción de tratamiento. Se logra tener buen control del movimiento distal del molar. Además, por ser un aparato fijo facilita al profesional alcanzar su objetivo en un menor tiempo.,4

La elección del tratamiento en el manejo integral del niño con pérdida prematura de espacio puede realizarse con un grupo interdisciplinario ${ }^{1,5,8}$ teniendo en cuenta las expectativas del paciente, así como sus necesidades funcionales y requisitos estéticos, para brindar a éste las condiciones adecuadas para el desarrollo y el mantenimiento del equilibrio entre la forma y la función del sistema estomatognático. ${ }^{1,2,8,14,16}$

\section{BIBLIOGRAFÍA}

1. Barba A, García N. Distalización de molares con Botón de Nance Modificado y Resorte Abierto de Nitinol. Revista Ortousta 2003; 3: 7 - 12.

2. Linden VD. As conseqüencias das perdas prematuras dos dentes deciduos. En: Ortodontia: Desenvolvimento da denticao. Quintessence Editora Ltda, 1ra. Ed. Sao Paulo. 1986: 129 - 153.

3. Lima SR, Panhóca HV. Barra transpalatina - aplicação e seus efeitos. Straight-wire 1994; 5: 13 - 21.

4. Ramos AL, Sakima MT, Santos A, Parsekian L, Ravelli B. Barra Platina. Dental Press Ortodont Ortop Facial 2000; 5. 75 - 85.

5. Graber TM. Vanarsdal RL. Ortodoncia: Principios generales y técnicas. Editorial Panamericana, 3ra. Ed., 2000: 523-528.

6. Proffit WR. Ortodoncia teoría y práctica. Mosby, 2da. Ed., Madrid; 2000: $128-133$.

7. Cañón OL, Zableh ME. Influencia del medio ambiente en la etiología de las maloclusiones: Primera parte. Revista Ortousta 2003; 3: 39 - 47.

8. Cárdenas D. Manejo y control de espacios. En: Fundamentos de Odontología. Odontología Pediátrica. Medellín, 1996: 217 - 248.

9. Moyers RE. Manual de ortodoncia. Editorial Panamericana, 4ta. Ed., Argentina. 1988.

10. Hixon EH. Oldfather RE. Estimation of the sizes of unerupted cuspid and bicuspid teeth. Am J Ortho Dentofac Orthop 1958.

11. McNamara J. A method of cefhalometric evaluation. Am J Ortho Dentofac Orthop 1984; 449-469.

12. Steiner $C$. The use of cefhalometrics as an aid to planning orthodontic treatment. Am J Ortho 1960; 46: 721 - 735.

13. Barba A. Una Alternativa para Controlar la Extrusión del Molar Superior. Revista Ortousta 2002; 1: 6-9.

14. Rivera M. Reyes M. Mendoza C. ¿Que aporta la terapia miofuncional al trabajo del ortodoncista? Revista Ortousta 2002; 2: 94 - 96.

15. Cañón OL, Ramos VM. El aparato craneomaxilar en tratamiento de temprano de la clase II división I. Revista Ortousta; 2003; 3: 21 - 28.

16. Gutiérrez VE. Efectividad de los magnetos en el tratamiento de maloclusión clase II. Revista Ortousta 2002; 2: $101-102$.

64 